DOI:

\title{
Efectos de una intervención de reducción del estrés basada en Mindfulness (MBSRP) sobre parámetros clínicos en la Artritis Reumatoidea
}

Graciela Castellano ORCID: 0000-0002-4381-007

Profesora Adjunta. Departamento Medicina Preventiva y Social. UdelaR.

Alicia Alemán. ORCID: 0000-0002-9247-1031

Profesora Agregada. Departamento Medicina Preventiva y Social. UdelaR.

Magdalena Isarri.

ORCID: 0000-002-0063-2011 Asistente. Departamento Medicina Preventiva y Social. UdelaR.

Walter Callero.

ORCID: 0000-0003-2122-4348

Asistente. Departamento Medicina

Preventiva y Social. UdelaR.

Juan Siri.

ORCID: 0000-0002-2687-406X

Asistente. Departamento Medicina

Preventiva y Social. UdelaR.

\author{
Effects of a Mindfulness-based stress reduction intervention (MBSRP) on \\ clinical parameters in Rheumatoid Arthritis \\ Efeitos de uma intervenção de redução do estresse baseada em \\ Mindfulness (MBSRP) sobre parâmetros clínicos na artrite reumatóide
}

Resumen: La artritis reumatoide es un trastorno autoinmune sistémico que causa inflamación extensa y persistente de las articulaciones. La inflamación persistente da lugar a muchas manifestaciones sistémicas y extraarticulares que afectan a la mayoría de los sistemas orgánicos, dando lugar a complicaciones graves y comorbilidades. El estrés psicológico es un factor provocador importante de los síntomas en las enfermedades inflamatorias crónicas. En los últimos años, el interés por abordar la respuesta al estrés a través de prácticas como la meditación ha aumentado asombrosamente, a pesar de la escasez de pruebas de que los beneficios reportados son específicos de esa práctica. La presente revisión sistemática se propone como objetivo evaluar la eficacia y seguridad de técnicas de meditación como tratamiento complementario de la AR. Se llevó a cabo una revisión sistemática de la literatura. Se consultaron las bases de datos Colaboración Cochrane, MEDLINE, Organización Mundial de la Salud, Plataforma de Registro Internacional de Ensayos clínicos para todos los ensayos registrados y en curso de realización y Clinicaltrials.gov Los datos extraídos se incluyeron en el kit de herramientas COVIDENCE y en el software REVMAN de la colaboración Cochrane. No pudo realizarse meta-análisis realizándose una síntesis narrativa de los resultados. Conclusiones: la técnica de Atención Plena (MBSR) es beneficiosa como tratamiento complementario de la Artritis Reumatoide, modificándose parámetros clínicos, pero con resultados heterogéneos en cuanto a los marcadores séricos de inflamación.

Palabras clave: Artritis reumatoide, estrés crónico, meditación.

Abstract: Rheumatoid arthritis is a systemic autoimmune disorder that causes extensive and persistent inflammation of the joints. Persistent inflammation leads to many systemic and extraarticular manifestations that affect most of the organ systems, leading to serious complications and comorbidities. Psychological stress is a major provocative factor of symptoms in chronic inflammatory diseases. In recent years, interest in addressing the stress response through practices such as meditation has increased dramatically, despite the paucity of evidence that the benefits reported are specific to that practice. The present systematic review aims to evaluate the effectiveness and safety of meditation techniques as a complementary treatment of RA. A systematic review of the literature was carried out. The databases Cochrane Collaboration, MEDLINE, World Health Organization, International Registry Platform of Clinical Trials for all the trials registered and in progress and Clinicaltrials.gov were consulted. extracted data were included in the COVIDENCE toolkit and in the REVMAN software of the Cochrane collaboration. Meta-analysis could not be performed, making a narrative synthesis of the results. Conclusions: the technique of Full Attention (MBSR) is beneficial as a complementary treatment of Rheumatoid Arthritis, modifying clinical parameters, but with heterogeneous results in terms of serum markers of inflammation.

Key words: Rheumatoid Arthritis, chronic stress, meditation. 
Resumo: A artrite reumatóide é um distúrbio auto-imune sistêmico que causa inflamação generalizada e persistente das articulações. A inflamação persistente leva a muitas manifestações sistêmicas e extra-articulares que afetam a maioria dos sistemas orgânicos, levando a sérias complicações e comorbidades. O estresse psicológico é um dos principais fatores desencadeantes dos sintomas nas doenças inflamatórias crônicas. Nos últimos anos, o interesse em abordar a resposta ao estresse por meio de práticas como a meditação aumentou dramaticamente, apesar da escassez de evidências de que os benefícios relatados são específicos dessa prática. A presente revisão sistemática tem como objetivo avaliar a eficácia e segurança das técnicas de meditação como tratamento complementar para AR. Uma revisão sistemática da literatura foi realizada. os bancos de dados Cochrane Collaboration, MEDLINE, Organização Mundial da Saúde, Plataforma de Registo Internacional de Ensaios Clínicos para todos os ensaios registrados e em andamento e consultou dados Clinicaltrials. gov extraído incluído no kit de ferramentas e Covidence no software REVMAN da colaboração Cochrane. Meta-análise não pôde ser realizada, fazendo uma síntese narrativa dos resultados. Conclusões técnica Mindfulness (MBSR) é benéfico como tratamento adjuvante de artrite reumatóide, modificando os parâmetros clínicos, mas com heterogénea em marcadores séricos de inflamação resulta.

Palavras-chave: Artrite reumatóide, estresse crônico, meditação.

Recibido: 04/10/2018 Aceptado: 28/04/2019

Departamento de Medicina Preventiva y Social. Facultad de Medicina. Universidad de la República (UdelaR). Montevideo, Uruguay.

Correspondencia: E-mail: sofiacastellano@gmail.com 


\section{Introducción}

La artritis reumatoide (AR) es un trastorno autoinmune sistémico que causa inflamación extensa y persistente del revestimiento sinovial de las articulaciones y vainas del tendón. La inflamación persistente da lugar a muchas manifestaciones sistémicas y extraarticulares que afectan a la mayoría de los sistemas orgánicos, dando lugar a complicaciones graves y comorbilidades tales como pulmón reumatoide, carditis, vasculitis, caquexia, anemia, aterosclerosis acelerada, enfermedad miocárdica y cerebrovascular, linfoma, osteoporosis y depresión. Las complicaciones y comorbilidades conducen a discapacidad, disfunción social y muerte prematura ${ }^{(1)}$. El tratamiento de la artritis reumatoide se centra en el manejo de síntomas como dolor, rigidez y movilidad limitada ${ }^{(2)}$.

La incidencia está relacionada con factores ambientales como el tabaquismo, las infecciones, las inmunizaciones, la obesidad y el estatus socioeconómico ${ }^{(3)}$.Fumar es el principal riesgo de desarrollar artritis reumatoide ${ }^{(4)}$.

La prevalencia mundial de la artritis reumatoide en 2010 se estimó en 0,24\% y fue aproximadamente dos veces más alta en las mujeres que en los hombres ${ }^{(5)}$. No ha habido ningún cambio perceptible en la prevalencia desde $1990{ }^{(5)}$. La prevalencia de la artritis reumatoide alcanza su punto máximo en la edad avanzada; se espera que el número de personas que viven con artritis reumatoide aumentara en el futuro. En Latinoamérica hay estudios de prevalencia realizados en Argentina y en Brasil donde ésta ha sido reportada en el 0,9 y el $0,45 \%$, respectivamente ${ }^{(6,7)}$. En Uruguay se estima que afecta a 35.000 personas, sobre todo a aquellas que se encuentran en la "etapa productiva" de sus vidas: la artritis afecta a personas en plena actividad laboral ${ }^{(7)}$.

\section{Diagnostico y evaluación de la Artritis Reumatoidea}

DAS 28 (Escala de actividad de la enfermedad, por sus siglas en inglés "Disease Activity Score"): Es un índice compuesto para valorar la actividad en la artritis reumatoide. Incluye un recuento de articulaciones dolorosas sobre 53 articulaciones, recuento de articulaciones tumefactas sobre 44 articulaciones, un reactante de fase (VSG) y la valoración global de la enfermedad por el paciente en una escala de 0-100. Estas variables se incluyen en una fórmula que pondera el valor de cada ítem. Rango: 1-9 (7).

Proteína C-reactiva (PCR): Esta prueba mide la cantidad de inflamación presente. Los niveles de PCR responden más rápidamente a los cambios en la actividad inflamatoria que la velocidad de eritrosedimentación. La PCR probablemente sea una medición precoz de la inflamación más sensible que la velocidad de eritrosedimentación ${ }^{(7)}$.

Factor Reumatoide (FR): El factor reumatoide es una prueba serológica inmunológica. Esta prueba a menudo (70 al $80 \%$ ) es positiva en personas con artritis reumatoide. Un resultado de más de $40 \mathrm{UI}$ pudiera considerarse anormal, los resultados pueden llegar hasta las $2000 \mathrm{UI}$ o más. Los análisis pueden ser negativos durante los primeros meses, haciendo la prueba menos útil para el diagnóstico temprano. EI FR a veces puede indicar la actividad de la enfermedad, aunque el FR no es específico de la artritis reumatoide y puede elevarse debido a una infección, gripe, etc ${ }^{(7)}$.

\section{Estrés crónico e inflamación}

El estrés psicológico es un factor provocador importante de los síntomas en las enfermedades inflamatorias crónicas. En los últimos años, el interés por abordar la respuesta al estrés a través de prácticas como la meditación ha aumentado asombrosamente, a pesar de la escasez de pruebas de que los beneficios reportados son específicos de esa práctica.

Desde 1980 a partir de los trabajos del Dr. Jon Kabat-Zinn fundador del Programa de Reducción del Estrés basado en Atención plena o Mindfulness (Mindfulness-Based Stress Reduction -MBSR), de la Universidad de Massachussetts, se viene desarrollando éste programa (8). Kabat-Zinn define a la atención plena como "prestar atención, en el momento presente, y sin prejuicios, al desarrollo de la experiencia momento a momento". El programa de reducción del estrés basado en Atención plena ${ }^{(8)}$ consta de una estructura y métodos que se describen en Anexo 1.

En un estudio realizado en el 2012, Rosenkranz y col. (9) compararon una intervención de reducción de estrés basada en la atención plena (MBSR, por sus siglas en inglés) de 8 semanas versus una intervención de control, el Programa de Mejoramiento de la Salud, en la capacidad de reducir el estrés psicológico y la inflamación inducida experimentalmente. 
La prueba de estrés social de Trier (TSST) se utilizó para inducir el estrés psicológico y la inflamación se produjo mediante la aplicación tópica de crema de capsaicina a la piel del antebrazo. Medidas inmunológicas y endocrinas de la inflamación y el estrés se recogieron antes y después de la intervención de MBSR. Los resultados muestran que los participantes asignados al azar a MBSR y al programa control tenían respuestas de cortisol evocadas por el estrés post-entrenamiento comparables, así como reducciones equivalentes en la angustia psicológica autoinformada y síntomas físicos. Sin embargo, el entrenamiento MBSR dio lugar a una respuesta inflamatoria post-estrés significativamente menor en comparación con el control, a pesar de los niveles equivalentes de hormonas de estrés. Estos resultados sugieren que las intervenciones conductuales diseñadas para reducir la reactividad emocional pueden ser de beneficio terapéutico en condiciones inflamatorias crónicas. Además, la práctica de la atención plena, en particular, puede ser más eficaz en el alivio de los síntomas que las actividades de promoción del bienestar cultivadas en el programa de mejoramiento de la salud (8).

Los objetivos del presente trabajo fueron evaluar la eficacia y seguridad de técnicas de meditación como tratamiento complementario de la Artritis Reumatoidea. Con este propósito se llevó a cabo una revisión sistemática de la literatura.

\section{Material y Métodos}

\section{A) Búsqueda y selección de estudios}

Se realizó una búsqueda sistemática en el mes de octubre del 2017, con los términos definidos en la pregunta de investigación (Anexo 2), en las siguientes bases de datos, con un período de búsqueda entre el 2007 y el 2017: Colaboración Cochrane, MEDLINE, Organización Mundial de la Salud, Plataforma de Registro Internacional de Ensayos clínicos para todos los ensayos registrados y en curso de realización, Clinicaltrials.gov (clinicaltrials.gov/).

\section{B) Extracción y análisis de los datos}

Los datos extraídos consistieron en: edad, proveniencia, porcentaje de mujeres en la población, empleo, historia de depresión, medicación utilizada para el tratamiento de la AR, duración en años de la enfermedad, indicadores clínicos de la enfermedad DAS 28, PCR en mg/ dl , Interleukina 6 , citokinaproinflamatoria y evaluación del dolor mediante escalas analógicovisuales (VAS-dolor, 0-100 y 0-20) . Además se evaluaron: presencia de angustia, bienestar, capacidad de atención plena y capacidad de afrontamiento.

La extracción de datos se realizó de manera independientemente por dos grupos de autores de revisión. Los datos extraídos se incluyeron en el kit de herramientas COVIDENCE y en el software REVMAN de la colaboración Cochrane, e incluyeron detalles del estudio, los criterios de inclusión y exclusión, los elementos de riesgo de sesgo, los datos sobre los participantes al inicio y los resultados con los datos correspondientes. Se evaluó la posibilidad de realizar metaanálisis.

\section{Resultados}

En la figura 1 se muestra el flujograma de selección de los estudios de la revisión. Fueron seleccionados 8 artículos por texto completo, para finalmente seleccionar 3 a los que se realizó el análisis. 


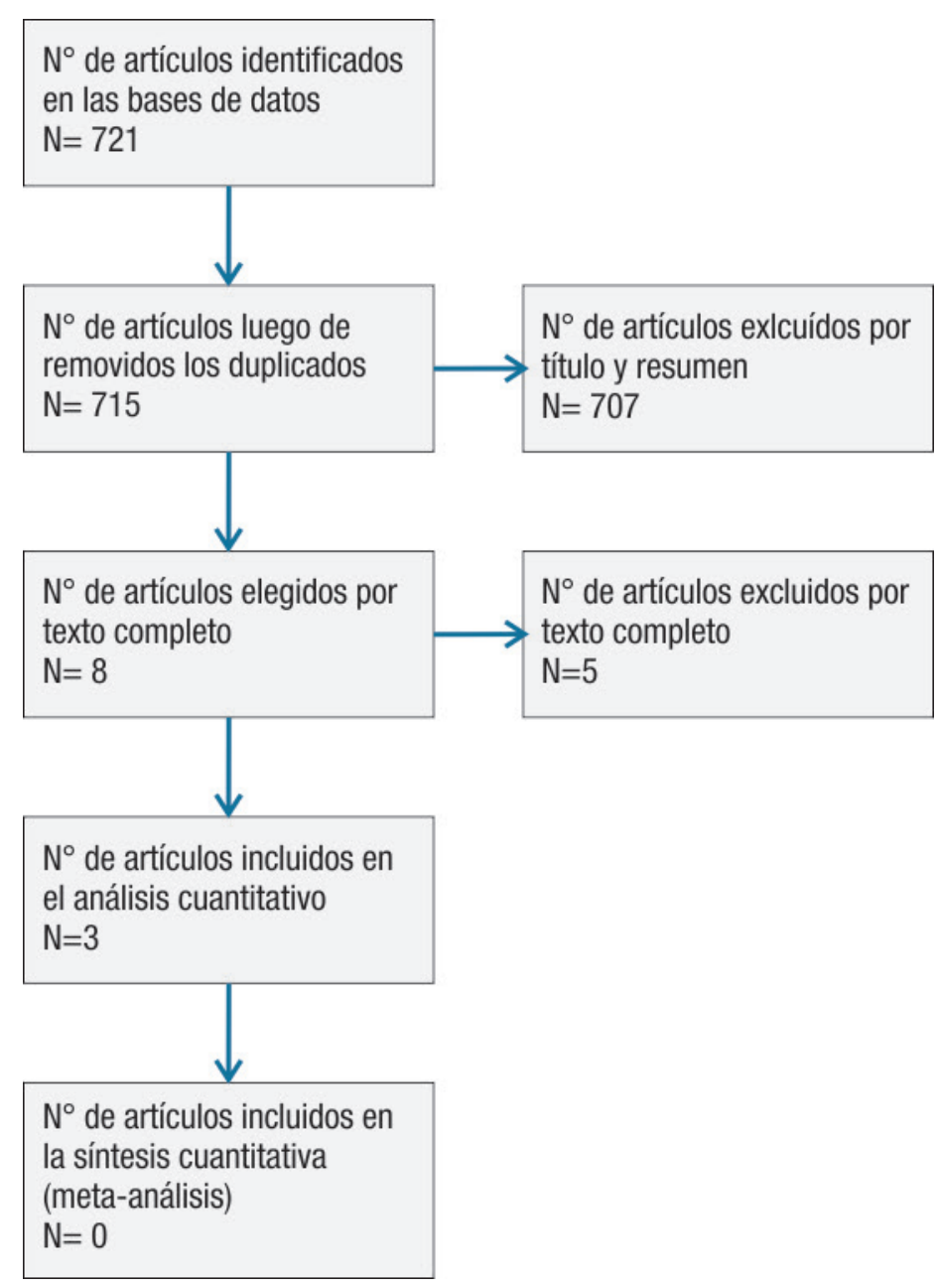

En la figura 2 se presentan los riesgos de sesgo promedio para todos los estudios.

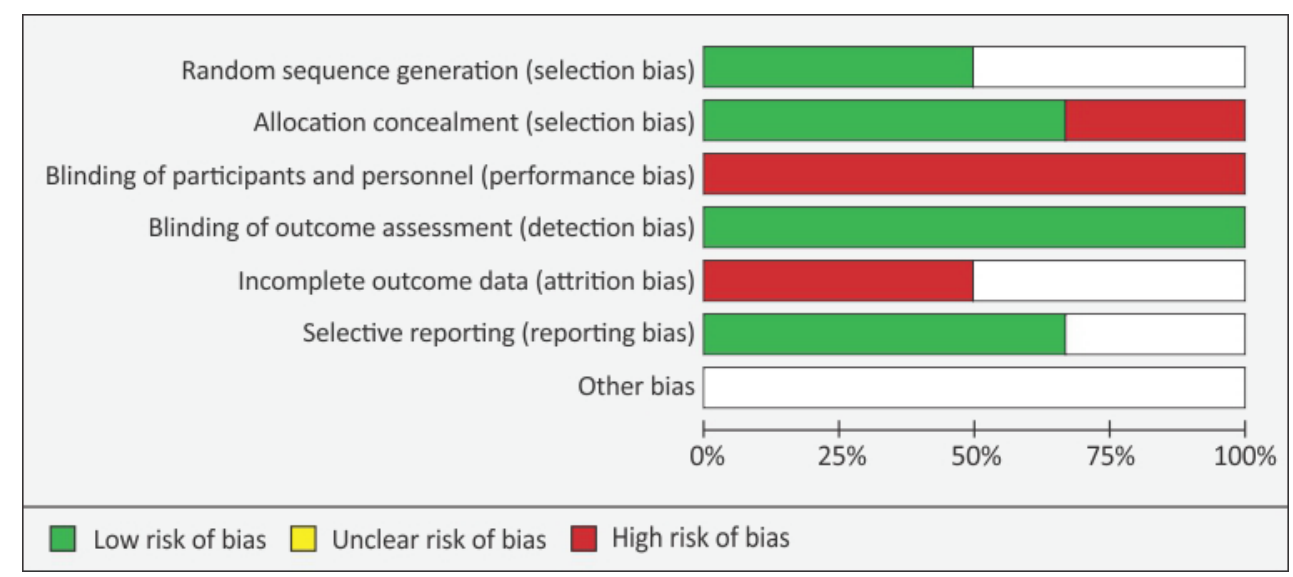

Dos autores utilizaron de forma independiente la herramienta Colaboración Cochrane "Riesgo de sesgo" para evaluar el riesgo de sesgo en los estudios seleccionados. Un tercer autor resolvió los desacuerdos en las calificaciones entre los autores.

Calificamos cada uno de los siguientes siete dominios como "bajo", "alto" o "poco claro":

1) generación de secuencia

2) ocultación de la asignación

3) cegamiento de los participantes y del personal

4) cegamiento de los evaluadores de resultados 
5) datos de resultados incompletos

6) presentación selectiva de resultados

7) otras fuentes de sesgo

Se utilizó la evaluación del riesgo de sesgo para juzgar la calidad de la evidencia para cada resultado de acuerdo con las recomendaciones de GRADE.

Las actividades relacionadas con la selección de estudios, extracción y manejo de datos, y la evaluación del riesgo de sesgo, se llevó a cabo utilizando el kit de herramientas COVIDENCE (Covidence 2016) y Revman V5.

Se realizó una síntesis narrativa de los hallazgos obtenidos. No pudo llevarse a cabo metaanálisis debido a la heterogenidad de las medidas de resultado en los estudios seleccionados.

En el estudio de Fogarty y cols, se evalúan 51 pacientes con AR, reclutados de dos hospitales públicos en Auckland, Nueva Zelanda. 26 y 25 participantes fueron aleatorizados a MBSR y grupos de control, respectivamente. Se realizaron evaluaciones a los 2, 4 y 6 meses de iniciada la intervención. La actividad de la enfermedad se evaluó usando la escala DAS28-CRP. Los componentes individuales del DAS28-CRP incluyen número de articulaciones doloridas e hinchadas, PCR y evaluación del dolor mediante una escala analógica visual de 0-100 mm. También se midió la duración de la rigidez matutina (minutos).

En el grupo MBSR, se produjo la mayor reducción en los puntajes DAS28-CRP, en comparación con el grupo control (Valor $p$ intervención $=0.01, \otimes 2=0,17$ ) inmediatamente después de la intervención y en ambos puntos de seguimiento. El grupo MBSR también mostró mejoras en la duración de la rigidez matutina (Valor $p=0.03$, $\nabla 2=0.13$ ) y puntuaciones de dolor (Valor $p=0.04, \nabla 2=0.10$ ). Estos efectos fueron evidentes después de la intervención y en ambos seguimientos en el tiempo.

En el estudio de Pradhan y cols, se evalúan síntomas depresivos, angustia psicológica, bienestar, atención plena, y actividad de la enfermedad (evaluada por DAS28).

Sesenta y tres participantes fueron asignados al azar: 31 a MBSR y 32 a control. A los 2 meses, no hubo diferencias significativas entre los grupos en cualquier resultado. A los 6 meses, hubo una mejora significativa en las variables angustia y bienestar (Valor $p=0.04$ y 0.03 , respectivamente), y una mejora marginalmente significativa en los síntomas depresivos y atención. Hubo una reducción del $35 \%$ en la angustia psicológica entre los tratados. La intervención no tuvo impacto en la actividad de la enfermedad.

El estudio de Zautra y cols, examina si la terapia cognitivo-conductual y las intervenciones de atención plena que se dirigen a las respuestas al estrés crónico, el dolor y la depresión, reducen el dolor y mejoran la calidad de la vida cotidiana para adultos con AR. Los 144 participantes se agruparon en grupos de $6-10$ participantes y fueron asignados aleatoriamente a 1 de 3 tratamientos: terapia cognitivo-conductual para el dolor $(\mathrm{P})$; meditación mindfulness y terapia de regulación de emociones (M); o grupo de solo educación (E), que sirvió como grupo control. Los autores evaluaron dolor auto-informado y niveles de interleuquina-6 (IL-6).

Los participantes que recibieron $\mathrm{P}$ mostraron una mejoría en el dolor autoinformado comparado con el grupo control y reducciones en el IL-6; ambos grupos $\mathrm{P}$ y $\mathrm{M}$ mostraron mejoras en la eficacia de afrontamiento respecto al grupo $\mathrm{E}$. El valor relativo de los tratamientos varió como una función de la historia de depresión. Los pacientes con depresión recurrente se beneficiaron más de $\mathrm{M}$ en varias medidas, especialmente las vinculadas al manejo emocional lo que indica que la regulación de la emoción fue un aspecto beneficioso para aquellos pacientes con características depresivas crónicas.

\section{Discusión y Conclusiones}

Los resultados obtenidos muestran que la técnica de Atención Plena (MBSR) es beneficiosa como tratamiento complementario de la Artritis Reumatoidea.

Un ensayo controlado aleatorio publicado por Cherkin y cols en 2016 (10), encontró que MBSR y la terapia cognitivo-conductual (TCC) produjeron alivio del dolor lumbar. Ambos fueron mejores que el cuidado habitual solo para aliviar el dolor y mejorar la función.

En los estudios comparativos seleccionados para el análisis de la revisión, la técnica MBSR mostró ser superior a otras intervenciones en la reducción del dolor, el control de la rigidez 
matutina, la angustia, síntomas depresivos, la atención y el afrontamiento. Respecto a la actividad de la artritis reumatoide los resultados obtenidos fueron heterogéneos.

Si bien en uno de los tres estudios se reportó una mejoría significativa de la actividad de esta enfermedad, en otro no se observó ninguna diferencia al respecto entre las distintas intervenciones. Asimismo no se detectaron diferencias significativas en los indicadores biológicos PCR y citokinaproinflamatoria (IL6). Los autores discuten los hallazgos relativos a la IL6, que van en contra de su hipótesis inicial, argumentando que la intervención M fue desarrollada para la intervención a diferencia de la terapia cognitivo conductual, en la que el equipo poseía mucha mayor experiencia.

Por su parte, Hilton y cols. publicaron en el 2017 (11), un metanálisis de 38 ensayos controlados aleatorios de meditación de atención plena y hallaron pruebas de mejora en la percepción del dolor, si bien la evidencia fue reportada como de calidad baja. También se encontraron efectos estadísticamente significativos para los síntomas de depresión y la calidad de vida, similar a lo hallado en esta revisión.

Por otra parte como limitaciones del estudio, debemos tener presente la heterogeneidad de las medidas de los resultados, razón por la cual, tuvimos que limitarnos a realizar una síntesis narrativa de los mismos y nos vimos imposibilitados de llevar a cabo un meta análisis.

De acuerdo a lo hallado, la intervención de reducción del estrés basada en Atención plena, parece contribuir favorablemente en los síntomas y signos de la enfermedad, así como en aspectos vinculados a la esfera emocional. Una posible explicación de estos hallazgos se propone en la vinculación entre el estrés crónico y la des-regulación de la respuesta inmunitaria. No es posible explicar la falta de variación en los marcadores de inflamación.

Sería importante ampliar y profundizar estudios que analicen la relación entre medidas biológicas e intervenciones reductoras del estrés mejor estandarizadas.

En conclusión si bien la MBSR parece ser una herramienta eficaz como tratamiento complementario de la Artritis Reumatoide aún hay evidencias limitadas al respecto. Es necesaria la realización de más estudios con mejor diseño para poder obtener evidencia científica de mayor calidad, que nos permitan confirmar su potencial aplicabilidad clínica. 


\section{Bibliografía}

1- McInnes IB, Schett G. The pathogenesis of rheumatoid arthritis.N Engl J Med. 2011;365(23):2205-19. doi: 10.1056/NEJMra1004965.

2- Aletaha D WM, Machold KP, Nell VP, Stamm T, Smolen JS: Remission and active disease in rheumatoid arthritis: defining criteria for disease activity states. . Arthritis Rheum. 2005 Sep;52(9):2625-36.

3- Myasoedova E, Crowson CS, Kremers HM, Therneau TM, Gabriel SE. Is the incidence of rheumatoid arthritis rising? Results from Olmsted County, Minnesota, 1955-2007.Arthritis Rheum. 2010 Jun;62(6):1576-82. doi: 10.1002/art.27425.

4- Scott DL WF, Huizinga TW.Rheumatoid arthritis.Lancet.2010, 9746(376):1094-1108.

5- Cross M SE, Hoy D, Carmona L, Wolfe F, Vos T, et al. The global burden of rheumatoid arthritis: estimates from the global burden of disease 2010 study. Ann Rheum Dis. 2014 Jul;73(7):1316-22. doi: 10.1136/annrheumdis-2013-204627

6- Scublinsky D VH, Citera G, Messina O, Scheines E, Rillo O, et al. The prevalence of rheumatoid arthritis in Argentina: A capture-recapture study in a city of Buenos Aires provinceJ ClinRheumatol. 2010;16(7):317-21. doi: 10.1097/RHU.0b013e3181f3bfdd.

7- http://reumauruguay.org/new/. Sociedad Uruguaya de Reumatologia 2015.

8- University of Massachusetts, Medical school. Mass: Programa de Reducción del Estrés basado en Atención Plena [en línea].2017[acceso: 23/02/2019] Disponible en:https://wwwumassmededu/cfm/ mindfulness-in-medicine/intro-to-mindfulness/

9- Rosenkranz MA, Maccoon DG, Sheridan JF, Kalin NH, Lutz A.A comparison of mindfulness-based stress reduction and an active control in modulation of neurogenic inflammation.Brain Behav Immun. 2013 Jan;27(1):174-84. doi: 10.1016/j.bbi.2012.10.013. 9

10- Cherkin DC, Sherman KJ, Balderson BH, Cook AJ, Anderson ML, Hawkes RJ, et al. Effect of Mindfulness-Based Stress Reduction vs Cognitive Behavioral Therapy or Usual Care on Back Pain and Functional Limitations in Adults With Chronic Low Back Pain: A Randomized Clinical Trial. JAMA. 2016;315(12):1240-9. doi: 10.1001/jama.2016.2323.

11- Hilton L, Hempel S, Ewing BA, Apaydin E, Xenakis L, Newberry S, et al. Mindfulness Meditation for Chronic Pain: Systematic Review and Meta-analysis. Ann BehavMed. 2017;51(2):199-213. doi: $10.1007 / \mathrm{s} 12160-016-9844-2$.

\section{Aporte cada autor al trabajo}

Graciela Castellano: $40 \%$

Alicia Alemán: $10 \%$

Magdalena Isarri: 30\%

Walter Callero: 15\%

Juan Siri: 5\% 


\section{Anexo 1}

a) Sesiones de Orientación Pre-Programa en Grupo (2.5 horas) seguidas de una breve entrevista individual (5-10 minutos)

b) Clases de ocho semanas (2.5-3.5 horas)

c) Un retiro de silencio durante todo el día durante la sexta semana del programa (7.5 horas).

d) Métodos "Formales" de meditación:

Escaneo del cuerpo - una meditación supina

Hatha Yoga suave - practicado con conciencia conciente del cuerpo

Meditación sentada - atención de la respiración, cuerpo, sentimientos, pensamientos, emociones, y la conciencia selectiva Meditación Caminando.

e) Prácticas de Meditación de la Atención Integral "Informales" (atención plena en la vida cotidiana):

Conciencia de acontecimientos agradables y desagradables

Conocimiento de la respiración

Conciencia deliberada de actividades y acontecimientos de rutina tales como: comer, conducir, caminar, conciencia de las comunicaciones interpersonales.

f) Actividades diarias a domicilio incluyendo un mínimo de 45 minutos por día de atención formal, 5-15 minutos de práctica informal, 6 días a la semana durante toda la duración del curso.

g) Instancias de diálogo individual y de grupo orientados a la reflexión sobre las tareas semanales. Una exploración de los obstáculos a la atención plena, al desarrollo y la integración de las habilidades y capacidades de autorregulación.

h) Incorporación de instrumentos de evaluación y autoevaluación de los participantes en la clase 8.

- Duración de las actividades en clase: 30 horas

- Total de tareas en casa: mínimo de 42-48 horas

- Orientación grupal: 2,5 horas

\section{Anexo 2}

1) "arthritis, rheumatoid"[MeSH Terms] OR ("arthritis"[All Fields] AND "rheumatoid"[All Fields]) OR "rheumatoid arthritis"[All Fields] OR ("arthritis"[All Fields] AND "rheumatoid"[All Fields]) OR "arthritis, rheumatoid"[All Fields]

2) mindfulness"[MeSH Terms] OR "mindfulness"[All Fields]

3) 1 and 2

4) randomizedcontrolled trial.pt.

5) controlledclinical trial.pt.

6) RandomizedControlledTrials/

7) RandomAllocation/

8) Double-BlindMethod/

9) Single-BlindMethod/

10) 4 or 5 or 6 or 7 or 8 or 9

11) 10 and 3 